BMJ

Open

Gastroenterology

\title{
Use of ColonFlag score for prioritisation of endoscopy in colorectal cancer
}

\author{
Ruth M Ayling (D , , A Wong, ${ }^{2}$ Finbarr Cotter ${ }^{3,4}$
}

To cite: Ayling RM, Wong A, Cotter F. Use of ColonFlag score for prioritisation of endoscopy in colorectal cancer. BMJ Open Gastro 2021;8:e000639. doi:10.1136/ bmjgast-2021-000639

Received 27 February 2021 Accepted 18 May 2021
Check for updates

(c) Author(s) (or their employer(s)) 2021. Re-use permitted under CC BY-NC. No commercial re-use. See rights and permissions. Published by BMJ.

${ }^{1}$ Clinical Biochemistry, Barts Health NHS Trust, London, UK ${ }^{2}$ Gastroenterology, Barts Health NHS Trust, London, UK ${ }^{3}$ Haemato-oncology, Barts Health NHS Trust, London, UK ${ }^{4}$ Joint NHS/academic appointment, Queen Mary University of London, London, UK

Correspondence to Ruth M Ayling; ruthayling@

clinicalbiochemistry.org.uk

\section{ABSTRACT}

Objective Colorectal cancer (CRC) is the fourth most common cancer in UK. Symptomatic patients are referred via an urgent pathway and although most are investigated with colonoscopy $<4 \%$ are diagnosed with cancer. There is therefore a need for a suitable triage tool to prioritise investigations. This study retrospectively examined performance of various triage tools in patients awaiting investigation on the urgent lower gastrointestinal cancer pathway

Design All patients over 40 years of age on the urgent pathway awaiting investigation for suspected CRC on 1 May were included. After 6 months, outcomes were evaluated and the performance of the faecal immunochemical test (FIT), faecal haemoglobin concentration, age and sex test (FAST) and the artificial intelligence algorithm ColonFlag were examined.

Results 532 completed investigations and received a diagnosis; 15 had CRC. 388 had a valid FIT result, of whom 11 had CRC; FAST Score $\geq 4.5$ had sensitivity of $72.7 \%$, specificity of $80.6 \%$ and would have failed to detect three tumours. Faecal haemoglobin (f-Hb) at cut-off of $10 \mu \mathrm{g} / \mathrm{g}$ and ColonFlag had equal sensitivity of $81.82 \%$, ColonFlag had greater specificity $73.47 \%$, compared with $64.99 \%$. Both tests would have failed to detect two tumours but not in the same patients; when used in combination, sensitivity and specificity were $100 \%$ and $49.4 \%$. When ColonFlag was applied to the cohort of 532 , an additional four tumours would have been detected in patients without a valid FIT.

Conclusion This study showed Colonflag to have equal sensitivity and greater specificity than $\mathrm{f}-\mathrm{Hb}$ at a cut-off of $10 \mu \mathrm{g} / \mathrm{g}$ as a triage tool for CRC

\section{INTRODUCTION}

Colorectal cancer (CRC) is the fourth most common cancer in the UK, with approximately 43000 patients diagnosed each year and the second largest cause of cancer death. ${ }^{1}$ To assist early detection, dedicated urgent pathways were established in England in 2000 to facilitate symptomatic patients receiving specialist assessment within 2 weeks if cancer was suspected. This pathway was revised with the provision of the NG12 guidance by the National Institute for Health and Care Excellence (NICE); this suggests a positive predictive value (PPV) of $3 \%$ as a threshold for urgent referral. ${ }^{2}$ In 2017, NICE issued diagnostics guidance $(\mathrm{DG} 30)^{3}$ on

Summary box
What is already known about this subject?
There is a need for suitable triage tools to pri-
oritise colonoscopy for colorectal carcinoma.
Measurement of faecal haemoglobin (f-HB) using
the faecal immunochemical test is used extensive-
ly for screening and assessment of symptomatic
patients. However, some tumours may give a false
negative result which may be more common with
right-sided lesions.
What are the new findings?
In this study, ColonFlag, an artificial intelligence
learning algorithm based on full blood count param-
eters, age and sex, was shown to have equal sensi-
tivity and better specificity than f-Hb at a cut-off of
10 1 gg/g for detection of colorectal carcinoma.
How might it impact on clinical practice in the
foreseeable future?
Further studies are required, but ColonFlag has
potential to be used alone or with f-Hb to improve
detection of colorectal cancer in symptomatic pa-
tients and possibly in screening programmes. As it
is based on the full blood count, it could be embed-
ded into laboratory computer systems to assist case
finding in primary and secondary care.

measurement of faecal haemoglobin (f-Hb) using faecal immunochemical testing (FIT) and updated NG12 to incorporate a recommendation for its measurement in patients with unexplained abdominal symptoms, without rectal bleeding, who do not meet criteria for urgent referral for suspected CRC. A f-Hb $\geq 10 \mu \mathrm{g}$ haemoglobin/g faeces $(\mu \mathrm{g} / \mathrm{g})$ was recommended as the cut-off to prompt urgent referral. The use of FIT in line with DG30 was incorporated into our local urgent referral pathway from April 2019. ${ }^{4}$

In addition to the investigation of symptomatic patients, bowel cancer screening exists in many countries and a programme has been in place in England since 2006. Screening has been shown to lower CRC mortality and reduce the incidence of CRC, due mainly to detection and removal of adenomatous polyps. ${ }^{5}$ However, engagement in screening 
is not universal and, of relevance to our practice, has been shown to be lower in inner London than elsewhere in the country. ${ }^{6}$

While FIT is well established on the pathways in use for both symptomatic and asymptomatic patients, there is scope for additional triage tools and in this context, a number of CRC prediction models have been designed. Some of these incorporate clinical variables which could potentially be captured in a single consultation, but further work is needed to ensure robust validation. ${ }^{7}$ The faecal haemoglobin concentration, age and sex test (FAST) is a three-variable model for CRC based on $\mathrm{f}-\mathrm{Hb}$ concentration, age and sex. Two thresholds of 2.12 and 4.5 have been identified with $99 \%$ and $90 \%$ sensitivity, respectively, for CRC. ${ }^{8}$

Association between components of the full blood count (FBC) and the detection of CRC has been reported in the literature for many years. ${ }^{9}$ NICE guidance recommends urgent referral to a gastroenterologist of unexplained iron deficiency anaemia in men with $\mathrm{Hb}<120 \mathrm{~g} / \mathrm{L}$ and postmenopausal women with $\mathrm{Hb}<100 \mathrm{~g} / \mathrm{L} .^{10}$

Triage tools exist which incorporate FBC changes into risk scores, one such being ColonFlag (Medial EarlySign, Kfar Malal, Israel) which identifies patients of 40 years or older at risk of CRC using artificial intelligence (AI) learning using their age, sex and FBC parameters based on an ensemble of decision trees. The rationale of the tool is that patients develop subtle changes in multiple indices of the FBC before becoming symptomatic from CRC. Although the parameters themselves may remain within the reference range, if more than one FBC is available, such changes can be detected by the AI algorithm and flagged as a potential indicator of CRC. The model was developed using data from healthy Israelis and CRC patients and trained using Israeli databases; validation was performed using additional cohorts within the USA and UK. ${ }^{11}$ Any number of FBCs can be used to derive the score, but a minimum of three over a period of 3-5 years is enough to reach almost optimal performance. We have previously reported its potential for use in triage of patients with anaemia. ${ }^{12}$

In the COVID-19 pandemic, England was placed into lockdown for the first time in March 2020 and nonemergency colonoscopy was temporarily suspended. During recovery from the first peak of infection, resumption of service was associated with reduced capacity because of the necessity for new health and safety protocols and with an accumulated backlog of patients requiring investigation. At this point, FIT was mandated as a criterion for referral. Some patients who had already been referred on our urgent pathway had not had the test performed in line with previous guidance and we co-ordinated a central process to offer them the test.

This study retrospectively investigated test performance of Colonflag for prioritisation in patients who had already been referred and were awaiting colonoscopy on 1 May in our hospital and compared it with the performance of FIT and FAST score.

\section{METHODS}

\section{Patients}

All adult patients over 40 years of age, referred to Barts Health NHS Trust on an urgent pathway with suspected CRC and awaiting investigation on 1 May 2020 were included in the study.

\section{Outcome definition}

After 6 months, clinical outcomes were collected and diagnoses of CRC, high risk adenomas (HRA) and inflammatory bowel disease (IBD) were confirmed from clinical notes, radiological reports and endoscopy and histological findings. HRA were defined using British Society of Gastroenterology guidance 2020. ${ }^{13}$

\section{Sample analysis}

Faecal samples were taken into a specimen collection device and returned to the Clinical Biochemistry department at Barts Health NHS Trust. They were stored at $4^{\circ} \mathrm{C}$ before analysis, which took place within 1 week of receipt and 2 weeks of sampling. The laboratory is accredited by the UK Accreditation Service to ISO 15189 standards. Analysis was performed using a single OC-SensoriO (Eiken Chemical Co., Tokyo, Japan). Inter-run imprecision was assessed with quality control materials (Eiken) in each run. Coefficients of variation were $2.8 \%$ at $14 \mu \mathrm{g} / \mathrm{g}$ and $3.0 \%$ at $91 \mu \mathrm{g} / \mathrm{g}$. External quality assurance was achieved via satisfactory performance in the relevant National External Quality Assurance Scheme. The lower limit of quantification was $4 \mu \mathrm{g} / \mathrm{g}$. The upper analytical limit was $200 \mu \mathrm{g} / \mathrm{g}$ and samples with a concentration above this were not diluted and reassayed but reported as $>200 \mu \mathrm{g} / \mathrm{g}$. If a patient returned more than one FIT sample, only the first test result was selected for inclusion in the analysis. FBCs were measured on a Sysmex XE 2100 (Sysmex, Milton Keynes, UK).

\section{Calculation of risk scores}

FAST score was calculated using the equation $\mathrm{f}-\mathrm{Hb}$ score $+(0.031 \mathrm{x}$ age in years $)+0.479$ if male. The $\mathrm{f}-\mathrm{Hb}$ score is 0 if the concentration is $0 \mu \mathrm{g} / \mathrm{g}, 0.6841$ if $1-19 \mu \mathrm{g} / \mathrm{g}, 2.824$ if $20-199 \mu \mathrm{g} / \mathrm{g}$ and $\geq 200 \mu \mathrm{g} / \mathrm{g} 4.184 .4 \mu \mathrm{g} / \mathrm{g}$ was taken to be the lower limit of quantification.

ColonFlag was calculated by Medial EarlySign. The parameters from all available FBCs from 2015 onwards were used, as these were easily accessible from current laboratory records. FBC parameters, age and sex were used to assign an individual risk score for each patient and to place them in one of four bands (high to low: bands 3 to 0 ) indicating their likelihood of CRC. The highest scoring band equated to a theoretical PPV of about $10 \%$.

\section{Statistical considerations}

Data were summarised and tabulated; population characteristics were summarised by appropriate descriptive statistics by data type.

Continuance measures (eg, sensitivity, specificity, PPV and NPV) were described by average and the exact $95 \%$ CI for each measure. 


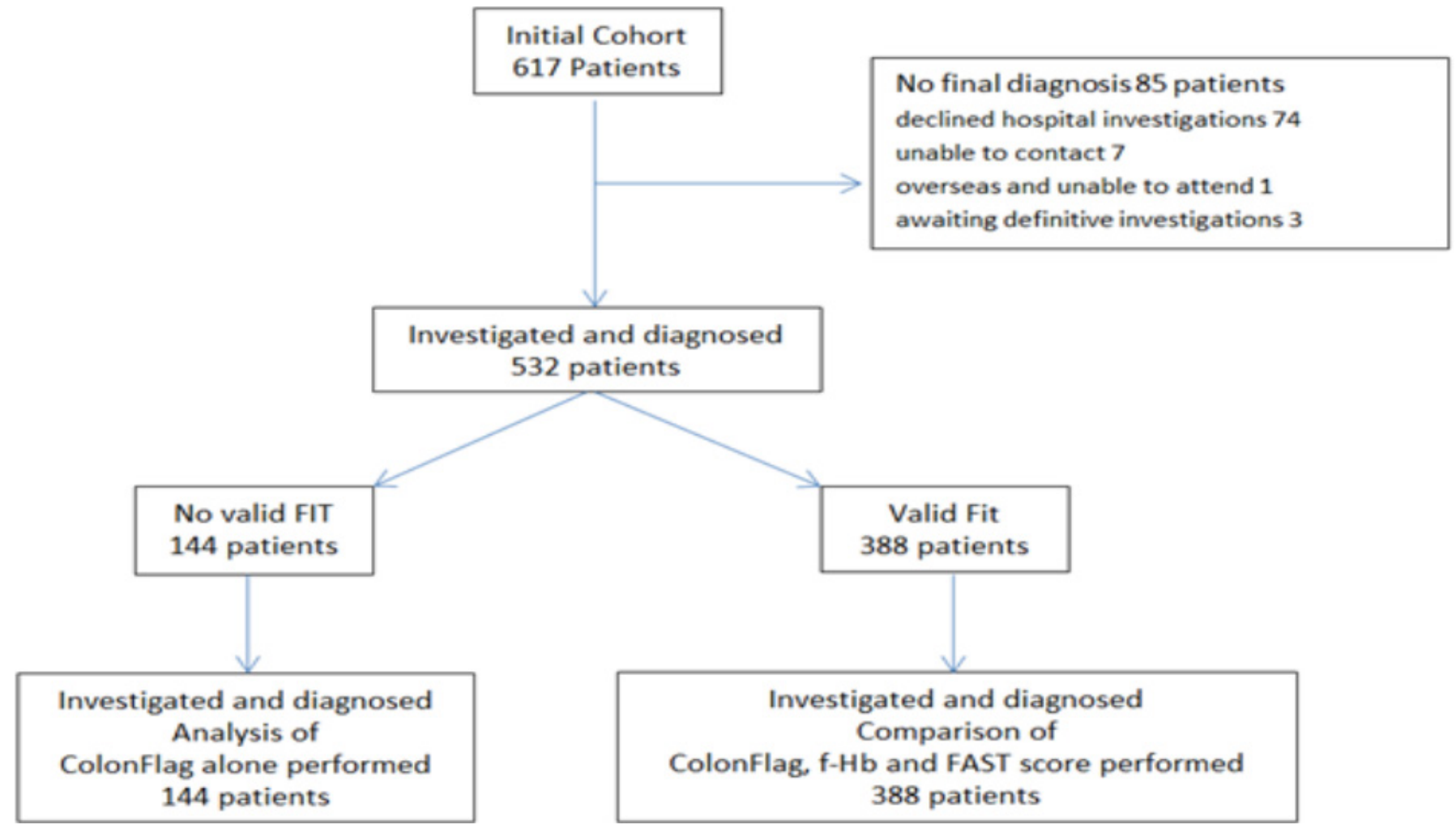

Figure 1 Study flow chart. f-HB, faecal haemoglobin; FIT, faecal immunochemical test.

Comparison for categorical parameters was tested by $\chi^{2}$ or Fisher's exact test (in case of low frequency). A calculated p-value of $5 \%$ or less was considered as statistically significant.

\section{Ethical considerations}

Data were gathered during routine patient care, therefore, ethical approval was not required. In order to mitigate risks associated with privacy, the data were provided to Medial EarlySign in de-identified format.

\section{RESULTS}

Data were obtained from 617 patients, $314(50.81 \%)$ male. Their median age was 63 years (range $40-98$ ). The study flow chart is shown in figure 1.

\section{Further investigation}

Investigations were performed on clinical grounds and a final diagnosis was obtained in 532 patients: 316 (59.4\%), underwent colonoscopy. 153 (28.8\%) abdominopelvic CT (with flexible sigmoidoscopy in addition in 15 and proctoscopy in six), $54(10.0 \%)$ CT colonography (with flexible sigmoidoscopy in addition in five) and six (1.1\%) flexible sigmoidoscopy alone. The patients who underwent colonoscopy were younger than those who underwent other definitive investigations (median 60.94 years, vs 67.21 years, $\mathrm{p}<0.001$ ) The others were not specifically investigated on the urgent pathway as they were under the care of other teams-one because he underwent emergency surgery for intussusception before investigations could be performed, one with known metastatic carcinoma of the prostate and one woman with iron deficiency anaemia and menorrhagia, 74 patients declined investigations, one was overseas and unable to attend and seven were unable to be contacted, despite multiple attempts.

\section{Final diagnoses}

On the basis of these investigations, 17 of the 532 patients $(3.2 \%)$ were found to have CRC, $28(5.2 \%)$ patients had HRA and $10(1.9 \%)$ had IBD. Low risk adenomas were detected in 85 patients. Malignancy was diagnosed in an additional nine patients (lung, breast, cervix (2), prostate, kidney (2), unknown primary and caecal neuroendocrine tumour). Of the 388 patients in whom a FIT test was performed and a final diagnosis available, $11(2.9 \%)$ were found to have CRC, 24 (6.3\%) HRA and 8 (2.1\%) IBD.

\section{Haemoglobin concentration}

The median blood haemoglobin $(\mathrm{Hb})$ concentration in men was $134 \mathrm{~g} / \mathrm{L}$ (range 68-182) and in women $122 \mathrm{~g} / \mathrm{L}$ (range 73-167). 92 men had a $\mathrm{Hb}<120 \mathrm{~g} / \mathrm{L}$ and 30 women older than 50 years had a $\mathrm{Hb}<100 \mathrm{~g} / \mathrm{L}$. In the group with a valid FIT, of the 11 patients with CRC four men were anaemic $(\mathrm{Hb}<130 \mathrm{~g} / \mathrm{L})$ and three women (haemoglobin $<120 \mathrm{~g} / \mathrm{L}$ ).

\section{Faecal immunochemical test results}

Of all the patients on the waiting list on 1 May, FIT had only been requested from $440(71.3 \%)$ and, of these samples, only 427 met preanalytical criteria for analysis. There was no significant difference in age and sex 
between those who had and had not provided a sample (p: 0.82 and 0.10 , respectively).

Of the 532 patients in whom investigation was performed and a final diagnosis available, 388 had performed a FIT and the $\mathrm{f}-\mathrm{Hb}$ concentrations were $<4 \mu \mathrm{g} / \mathrm{g}$ in $134(34.5 \%)$, $<10 \mu \mathrm{g} / \mathrm{g}$ in $247(63.7 \%), \geq 10-99 \mu \mathrm{g} / \mathrm{g}$ in $94(24.2 \%)$ and $\geq 100 \mu \mathrm{g} / \mathrm{g}$ in $47(12.1 \%)$

\section{FAST score}

Of the 388 patients with a final diagnosis who provided a FIT sample, 312 had a FAST score above 2.12 and 81 had a score above 4.5.

\section{ColonFlag}

.Using ColonFlag, patients were divided into one of four bands indicating their likelihood of CRC. Of those in whom a final diagnosis was made 165 patients $(31.3 \%)$ were in band 3 (highest risk), $111(21.0 \%)$ in band 2, $128(24.2 \%)$ in band 1 and $128(24.2 \%)$ in band 0 . The distribution of banding in the subset of 388 patients who provided a FIT sample was as follows band 3109 (28.1\%), band $285(21.9 \%)$, band $194(24.2 \%)$ and band 0100 (25.8\%) patients. The median number of FBC available was eight (range 1-85). In the group of 388 patients, 34 had less than three FBC and of these, 17 had only one. Of the 11 patients in this group with CRC, the scoring was on 5-57 measurements.

Table 1 shows the performance of FIT, ColonFlag and FAST score in diagnosis of CRC, HRA and IBD in the 388 patients in whom a viable FIT was available. FAST score at a cut-off of 4.15 was the least sensitive test for detection of CRC but had the highest specificity and would have detected eight tumours with only 81 patients $(20.9 \%)$ requiring colonoscopy. Using a cut-off of 2.12 would have detected all tumours but reduced specificity to $20.16 \%$ and would have required colonoscopy in $80 \%$. At a cutoff of $10 \mu \mathrm{g} / \mathrm{g}, \mathrm{f}-\mathrm{Hb}$ failed to detect two tumours. One of these patients had a T3 tumour of the caecum and a $\mathrm{f}-\mathrm{Hb}$ concentration of $4 \mu \mathrm{g} / \mathrm{g}$, the other had a T4 tumour of the caecum and $\mathrm{f}-\mathrm{Hb}$ concentration of $7 \mu \mathrm{g} / \mathrm{g}$. Both of these patients were detected by ColonFlag at band 3 . Two different patients were not detected by ColonFlag at band 3. One had a banding of $2, \mathrm{f}-\mathrm{Hb}$ of $78 \mu \mathrm{g} / \mathrm{g}$ and a T4 tumour in the transverse colon. The other patient had a banding of $1, \mathrm{f}-\mathrm{Hb}$ of $78 \mu \mathrm{g} / \mathrm{g}$ and a $\mathrm{T} 4$ tumour in the caecum. Using $\mathrm{f}-\mathrm{Hb}$ at a cut-off of $10 \mu \mathrm{g} / \mathrm{g}$ as a triage tool would have required colonoscopy in 141 $(36.3 \%)$ of the 388 patients, using ColonFlag at band 3 would have required colonoscopy in 109 patients (28\%). Both methods would have detected 9 of 11 tumours. The combination of either, or both, of f-Hb at a cut-off of $10 \mu \mathrm{g} / \mathrm{g}$ and ColonFlag band 3, would have detected two more cancers and three more HRA than FIT alone.

In the whole cohort of 532 patients, including those with no viable FIT sample, triage using ColonFlag at band 3 would have led to 165 (31\%) colonoscopies being prioritised with detection of 15 of 17 tumours and would have detected four tumours in patients in whom a $\mathrm{f}-\mathrm{Hb}$ result was not available; the test performance being sensitivity $88.24 \%$ (95\% CI: 63.56 to 98.54 ), specificity $71.07 \%-95 \%$ CI: 66.94 to 74.94 ), PPV 9.15\% (95\% CI: 7.47 to 11.15 ) and NPV $99.45 \%$ (95\% CI: 98.03 to 99.85 ).

\section{DISCUSSION}

In this study, we retrospectively investigated the potential use of ColonFlag to prioritise patients awaiting colonoscopy during the COVID-19 pandemic. In our cohort of 532 patients, triage according to the highest risk band 3 would have led to $165(31 \%)$ colonoscopies being prioritised with detection of 15 of 17 tumours. ColonFlag differs from other risk-prediction models in that it has the ability to test people automatically using routinely available laboratory data and we would have enabled us to detect four tumours in patients who did not have a $\mathrm{f}-\mathrm{Hb}$ result available.

Measurement of $\mathrm{f}-\mathrm{Hb}$ using FIT was recommended by NICE to assist with triage of patients deemed to be at low risk of CRC, using a cut-off of $10 \mu \mathrm{g} / \mathrm{g}$ (NG12). However, the test has been widely investigated and is now used in patients at both low $^{4}$ and high ${ }^{14}$ risk of CRC. Previous studies investigating the test performance of $\mathrm{f}-\mathrm{Hb}$ in CRC have found sensitivities between $83.3 \%$ and $90.9 \%$ at a cutoff of $10 \mu \mathrm{g} / \mathrm{g},{ }^{15-17}$ comparable to our findings. Specificities in these studies were slightly higher at $79.1 \%-83.5 \%$, but this may reflect a difference in population as our patients were referred as the start of the pandemic which may not represent a typical sample. In a study of 755 patients, $\mathrm{f}-\mathrm{Hb}$ at a cut-off of $\geq 10 \mu \mathrm{g} / \mathrm{g}$ was shown to be an objective predictor of underlying pathology when used at point of referral with sensitivity of $68.6 \%$, specificity of $83.6 \%$, PPV of $39.8 \%$ and NPV of $94.4 \% .{ }^{16}$ Our results add further to weight to these findings and show potential advantage of FIT in combination with ColonFlag. Both tests are reliant on blood loss from colonic lesions to provide parameters for indication of risk. For FIT, this is direct measurement of haemoglobin in faeces but with ColonFlag, it is the result of subtle changes in FBC parameters.

FAST score has been proposed as a tool to assist with prioritisation of referral of patients from primary care and for segregation into a high risk group in which $90 \%$ of the CRC would be expected, an intermediate risk group where an additional 9\% of CRC might be found and a third group hypothetically containing the remaining $1 \%$ of CRC. ${ }^{8}$ Our results show sensitivities of 100\% (95\% CI: 71.51 to 100.00 ) and $72.73 \%$ (95\% CI: 39.03 to 93.98 ) at the 2.12 and 4.5 cut-offs. We used the previously described formula for $\mathrm{FAST}^{18}$ related to the limit of quantification of our system of $\mathrm{f}-\mathrm{Hb}$ of $4 \mu \mathrm{g} / \mathrm{g}$. It has been suggested that for optimal test performance, different analytical systems may require tailoring of the formula. While we found FAST to be the least sensitive test for detection of CRC, its high specificity could be of value in situations where colonoscopy provision is temporarily restricted such as the early recovery phase after 
Table 1 Comparison of the performance of triage tools in the detection of colorectal cancer and significant bowel disease in cohort of 388 patients in whom faecal immunochemical testing was performed

$\mathrm{f}-\mathrm{Hb} \geq 10 \mu \mathrm{g} / \mathrm{g}$ and/

or

FAST score $\geq 4.5 \quad \mathrm{f}-\mathrm{Hb} \geq 10 \mu \mathrm{g} / \mathrm{g} \quad$ Colon flag band $3 \quad$ ColonFlag band 3

\begin{tabular}{|c|c|c|c|c|}
\hline \multicolumn{5}{|l|}{ CRC } \\
\hline No of cases & 11 & 11 & & 1 \\
\hline True positives & 8 & 9 & 9 & 11 \\
\hline True negatives & 303 & 245 & 277 & 187 \\
\hline False positives & 73 & 132 & 100 & 190 \\
\hline False negatives & 3 & 2 & 2 & 0 \\
\hline Sensitivity & $72.7(39.0-94.0)$ & $81.8(48.2-97.7)$ & $81.8(48.2-97.7)$ & $100(71.5-100)$ \\
\hline Specificity & $80.6(76.2-84.5)$ & $64.9(59.9-69.8)$ & $73.5(68.7-77.9)$ & $49.6(44.4-54.8)$ \\
\hline PPV & $9.9(6.7-14.3)$ & $6.4(4.8-8.5)$ & $8.3(6.1-11.1)$ & $5.5(4.9-6.0)$ \\
\hline NPV & $99.0(97.5-99.6)$ & $99.2(97.2-99.8)$ & $99.3(97.6-99.8)$ & 100 \\
\hline \multicolumn{5}{|l|}{$\mathrm{CRC}+\mathrm{HRA}$} \\
\hline No of cases & 35 & 35 & 35 & 35 \\
\hline True positives & 21 & 25 & 15 & 30 \\
\hline True negatives & 293 & 237 & 259 & 182 \\
\hline False positives & 60 & 116 & 94 & 171 \\
\hline False negatives & 14 & 10 & 20 & 5 \\
\hline Sensitivity & $60.0(42.1-76.1)$ & $71.4(53.7-85.4)$ & $42.9(26.3-60.7)$ & $85.7(69.7-95.2)$ \\
\hline Specificity & $83.0(78.7-86.8)$ & $67.3(62.2-72.2)$ & $73.4(68.4-77.9)$ & $51.6(46.2-56.9)$ \\
\hline PPV & $25.9(19.70-33.3)$ & $17.9(14.4-21.0)$ & $13.7(9.5-19.5)$ & $14.9(12.9-17.3)$ \\
\hline NPV & $95.4(93.3-96.9)$ & $95.6(93.3-97.6)$ & $92.8(90.6--94.6)$ & $97.3(94.1-98.8)$ \\
\hline \multicolumn{5}{|l|}{$\mathrm{CRC}+\mathrm{HRA}+\mathrm{IBD}$} \\
\hline No of cases & 43 & 43 & 43 & 43 \\
\hline True positives & 29 & 33 & 17 & 38 \\
\hline True negatives & 293 & 237 & 253 & 182 \\
\hline False positives & 52 & 108 & 92 & 163 \\
\hline False negatives & 14 & 10 & 26 & 5 \\
\hline Sensitivity & $67.4(51.5-80.9)$ & $76.7(61.4-88.2)$ & $39.5(25.0-55.6)$ & $88.4(74.9-96.1)$ \\
\hline Specificity & $84.9(80.7-88.5)$ & $68.7(63.5-73.6)$ & $73.3(68.3-78.0)$ & $52.8(47.3-58.1)$ \\
\hline PPV & $35.8(28.7-43.6)$ & $23.4(19.6-27.7)$ & $15.6(10.9-21.8)$ & $18.9(16.64-21.4)$ \\
\hline NPV & $95.4(93.1-97.0)$ & $96.0(93.2-97.6)$ & $69.6(64.7-74.1)$ & $97.3(94.1-98.8)$ \\
\hline
\end{tabular}

CRC, colorectal cancer; f-HB, faecal haemoglobin; HRA, high risk adenomas; IBD, inflammatory bowel disease; NPV, negative predictive value; PPV, positive predictive value.

interruption of service as occurred at the time that this study was performed.

We accept that our study is small, the cohort containing only 11 patients with CRC and that further work is required, but hypothesise that ColonFlag is potentially of value as a triage tool. It differs from other risk-prediction models in that it has the ability to test people automatically using routinely available laboratory data. Reluctance of our patients to complete FIT and to undergo invasive investigations and a reduction of colonoscopy provision in the current climate resulting from COVID-19 are drivers for consideration of an AI triage tool based on the parameters of the FBC. ColonFlag has the potential to be embedded into hospital computer systems in order to generate a prediction score associated with existing FBC analyses and patients' scores could be automatically updated with each FBC and to assist case finding in various contexts. Approaching the test from the angle of high specificity, patients with a raised or increasing score could be highlighted in order to be considered for investigation. Alternatively, at lower cut offs, where sensitivity is greater, the algorithm might be of benefit to target patients who have not engaged with bowel screening services. Uptake of faecal testing is not universal, in screening programmes or patients with symptoms and $20 \%$ of CRC still present through Emergency pathways. ${ }^{19}$ 
This has multiple causes including cultural and health inequalities related to deprivation. ${ }^{20}$ Optimal performance of ColonFlag requires prior engagement by the population to have had blood tests and serial results may be least accessible in those people who decline faecal testing. However, potentially the use of ColonFlag as a test in iron deficiency ${ }^{12}$ or as part of routine hospital admissions in the CRC screening age group could assist both with early detection and reduction of the mortality associated with late presentation.

In our cohort, both $\mathrm{f}-\mathrm{Hb}$ at a cut-off of $10 \mu \mathrm{g} / \mathrm{g}$ and ColonFlag at band 3 failed to detect two patients, but these were not the same patients. Both the patients whose CRC was not detected by f-Hb using a cut-off of $10 \mu \mathrm{g} / \mathrm{g}$ had tumours located in the right side of the colon. It has been reported that $\mathrm{f}-\mathrm{Hb}$ is less accurate in detecting rightsided than left-sided CRC. ${ }^{21}$ Possible reasons suggested include right-sided lesions having potential to grow more rapidly and bleed less because of their specific phenotypic characteristics, less susceptibility to mechanical triggers exacerbating bleeding and a longer transit with more opportunity for degradation of haemoglobin.

In conclusion, we evaluated the performance of various tools to assist prioritisation for colonoscopy in an urgent lower gastrointestinal cancer pathway. We propose that AI based on FBC parameters using ColonFlag has potential both for prioritisation of symptomatic patients and to target patients who have not engaged with bowel screening services.

Contributors RMA and FC designed the study, AW and RMA collected the data, all authors were involved in writing of and approved the final version of the manuscript.

Funding The authors have not declared a specific grant for this research from any funding agency in the public, commercial or not-for-profit sectors.

Competing interests None declared.

Patient consent for publication Not required.

Provenance and peer review Not commissioned; externally peer reviewed.

Data availability statement All data relevant to the study are included in the article or uploaded as supplementary information. Deidentified participant data are stored securely by the authors and would potentially be available upon reasonable request.

Open access This is an open access article distributed in accordance with the Creative Commons Attribution Non Commercial (CC BY-NC 4.0) license, which permits others to distribute, remix, adapt, build upon this work non-commercially, and license their derivative works on different terms, provided the original work is properly cited, appropriate credit is given, any changes made indicated, and the use is non-commercial. See: http://creativecommons.org/licenses/by-nc/4.0/.

ORCID iD

Ruth M Ayling http://orcid.org/0000-0003-4662-2502

\section{REFERENCES}

1 Cancer Research UK. Bowel cancer incidence statistics, 2016. Available: http://www.cancerresearchuk.org/health-professional/ cancer-statistics-by-cancer-type/bowel-cancer/incidence [Accessed Feb 2021].

2 National Institute for Health and Care Excellence. Suspected cancer; recognition and referral (NG12), 2015. Available: www.nice.org/ guidance/ng12 [Accessed Feb 2020].

3 National Institute for Health and Care Excellence. Quantitative immunochemical tests to guide referral for colonoscopy in primary care. Diagnostics guidance [DG30], 2017. Available: https://www. nice.org.uk.guidance/dg30 [Accessed Feb 2021].

4 Ayling RM, Machesney M. Service evaluation of faecal immunochemical testing introduced for use in North East London for patients at low risk of colorectal cancer. J Clin Pathol 2021;74:163-6.

5 Navarro M, Nicolas A, Ferrandez A, et al. Colorectal cancer population screening programs worldwide in 2016: an update. World J Gastroenterol 2017;23:3632-42.

6 Logan RFA, Patnick J, Nickerson C, et al. Outcomes of the bowel cancer screening programme (BCSP) in England after the first 1 million tests. Gut 2012;61:1439-46.

7 Williams TGS, Cubiella J, Griffin SJ, et al. Risk prediction models for colorectal cancer in people with symptoms: a systematic review. BMC Gastroenterol 2016;16:63.

8 Cubiella J, Digby J, Rodríguez-Alonso L, et al. The fecal hemoglobin concentration, age and sex test score: development and external validation of a simple prediction tool for colorectal cancer detection in symptomatic patients. Int J Cancer 2017;140:2201-11.

9 Virdee PS, Marian I, Mansouri A. The full blood count test for colorectal cancer detection: a systematic review. meta-analysis and critical appraisal Cancers 2020;12:1238 http://doi.org

10 National Institute for Health and Care Excellence. Clinical knowledge summary. management of iron efficiency anaemia, 2018. Available: https://cks.nice.org.uk/topics/anaemia-iron-deficiency/management/ management/\#referral [Accessed Feb 2020].

11 Kinar Y, Kalkstein N, Akiva P, et al. Development and validation of a predictive model for detection of colorectal cancer in primary care by analysis of complete blood counts: a binational retrospective study. J Am Med Inform Assoc 2016;23:879-90.

12 Ayling RM, Lewis SJ, Cotter F. Potential roles of artificial intelligence learning and faecal immunochemical testing for prioritisation of colonoscopy in anaemia. Br J Haematol 2019;185:311-6.

13 Rutter MD, East J, Rees CJ, et al. British Society of Gastroenterology/Association of Coloproctology of great britain and Ireland/Public health England post-polypectomy and post-colorectal cancer resection surveillance guidelines. Gut 2020;69:201-23.

14 Chapman C, Thomas C, Morling J. Early clinical outcomes of a rapid colorectal cancer diagnosis pathway using faecal immunochemical testing in Nottingham colorectal dis 2020;22:679-88.

15 Laszlo HE, Seward E, Ayling R. Quantitative faecal immunochemical test for patients with high risk bowel symptoms; a prospective cohort study. medRxiv 2020.

16 Mowat C, Digby J, Strachan JA, et al. Faecal haemoglobin and faecal calprotectin as indicators of bowel disease in patients presenting to primary care with bowel symptoms. Gut 2016;65:1463-9.

17 D'Souza N, Georgiou Delisle T, Chen M, et al. Faecal immunochemical test is superior to symptoms in predicting pathology in patients with suspected colorectal cancer symptoms referred on a 2WW pathway: a diagnostic accuracy study. Gut 2021;70:1130-8.

18 Digby J, Strachan JA, Mowat C, et al. Appraisal of the faecal haemoglobin, age and sex test (fast) score in assessment of patients with lower bowel symptoms: an observational study. BMC Gastroenterol 2019;19:213.

19 Renzi C, Lyratzopoulos G, Card T, et al. Do colorectal cancer patients diagnosed as an emergency differ from non-emergency patients in their consultation patterns and symptoms? A longitudinal data-linkage study in England. Br J Cancer 2016;115:866-75.

20 Lal N, Singh HKSI, Majeed A, et al. The impact of socioeconomic deprivation on the uptake of colorectal cancer screening in London. $J$ Med Screen 2020:096914132091620.

21 Zorzi M, Hassan C, Capodaglio G, et al. Divergent long-term detection rates of proximal and distal advanced neoplasia in fecal immunochemical test screening programs: a retrospective cohort study. Ann Intern Med 2018;169:602-9. 